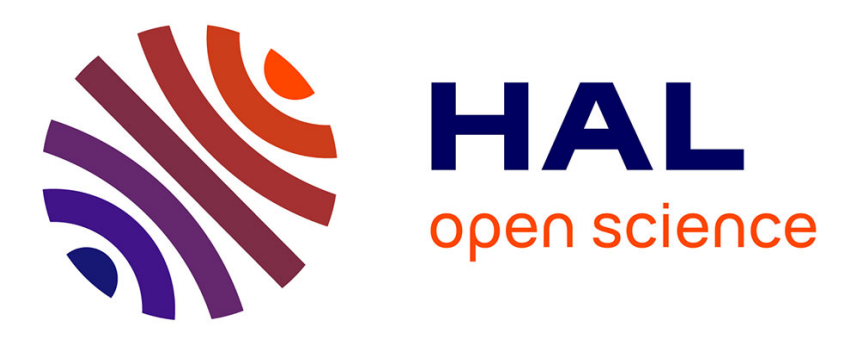

\title{
Amélioration de la sensibilité en spectroscopie de saturation hétérodyne grâce à l'utilisation de plusieurs classes de vitesses
}

G. Camy, R. Amer, N. Courtier

\section{- To cite this version:}

G. Camy, R. Amer, N. Courtier. Amélioration de la sensibilité en spectroscopie de saturation hétérodyne grâce à l'utilisation de plusieurs classes de vitesses. Revue de Physique Appliquée, 1984, 19 (8), pp.635-639. 10.1051/rphysap:01984001908063500 . jpa-00245232

\section{HAL Id: jpa-00245232 \\ https://hal.science/jpa-00245232}

Submitted on 1 Jan 1984

HAL is a multi-disciplinary open access archive for the deposit and dissemination of scientific research documents, whether they are published or not. The documents may come from teaching and research institutions in France or abroad, or from public or private research centers.
L'archive ouverte pluridisciplinaire HAL, est destinée au dépôt et à la diffusion de documents scientifiques de niveau recherche, publiés ou non, émanant des établissements d'enseignement et de recherche français ou étrangers, des laboratoires publics ou privés. 


\title{
Amélioration de la sensibilité en spectroscopie de saturation hétérodyne grâce à l'utilisation de plusieurs classes de vitesses $\left(^{*}\right)$
}

\author{
G. Camy, R. Amer (**) et N. Courtier \\ Laboratoire de Physique des Lasers (***), Université Paris-Nord, \\ avenue J.-B. Clément, 93430 Villetaneuse, France
}

(Reçu le 7 février 1984, révisé le 21 mai, accepté le 22 mai 1984)

\begin{abstract}
Résumé. - Dans le but d'améliorer la sensibilité de la détection en spectroscopie de saturation, nous proposons d'associer deux méthodes : la contribution de plusieurs classes de vitesses au signal de saturation et la spectroscopie hétérodyne à très haute résolution. Le principe consiste à moduler en phase (ou en fréquence, ou en amplitude) le faisceau de sortie d'un laser monomode stabilisé en fréquence, puis à utiliser ce faisceau modulé dans une expérience de spectroscopie de saturation hétérodyne. La comparaison des résultats obtenus dans une même expérience avec et sans modulation du faisceau met en évidence le gain en sensibilité obtenu par cette méthode. Cette amélioration provient du fait qu'en spectroscopie de saturation conventionnelle on sélectionne une seule classe de vitesses, alors que dans la méthode proposée plusieurs classes de vitesses contribuent au signal.
\end{abstract}

\begin{abstract}
In order to improve the detection sensitivity in saturation spectroscopy experiments we propose a method which combines the advantages of heterodyne saturation spectroscopy and of the contribution of several velocity classes to the signal. Its principle lies in a phase (or frequency, or amplitude) modulation of a monomode frequency stabilized laser beam, which is then used in a heterodyne saturation spectroscopy experiment. The experimental results compared to those obtained in saturated absorption with monochromatic light demonstrate an improvement of the sensitivity owing to the contribution of several velocity classes to the signal.
\end{abstract}

Dans les expériences habituelles de spectroscopie d'absorption saturée en cellule, le gain en résolution par rapport à l'absorption linéaire est lié à la sélection d'une seule classe de molécules : celle qui se propage perpendiculairement aux faisceaux lumineux. $\mathrm{Si}$ on désigne par $\mathrm{O} z$ la direction de propagation du faisceau saturant, on ne sélectionne donc que les molécules dont la composante de vitesse dans cette direction vaut $v_{z}=0$ à $\Delta v_{z} / 2$ près, avec $\Delta v_{z}=\Gamma / 2$, où $\Gamma$ est la largeur de raie.

Cette méthode permet de se soustraire à l'élargissement Doppler du $1^{\mathrm{er}}$ ordre, mais du même coup, elle n'autorise qu'une très faible fraction des molécules, de l'ordre de $\Gamma / \Delta \omega_{\mathrm{D}}$, à contribuer au signal, car dans tous les cas d'intérêt pratique, la largeur de raie $\Gamma$ est très faible devant la largeur Doppler $\Delta \omega_{\mathrm{D}}$.

Il est alors très tentant d'essayer d'améliorer le rapport signal/bruit en faisant contribuer au signal

(*) Travail effectué avec le soutien du Bureau National de Métrologie.

(**) Faculté des Sciences, Université d'Alexandrie, R.A.E.

${ }^{* * *}$ ) Laboratoire associé au C.N.R.S., L.A. 282. d'autres classes de vitesses que la classe $v_{z}=0$. Une première méthode a été proposée dans ce but dans les références $[1,2]$. Elle fait appel à un laser à colorant fonctionnant en modes bloqués. Un tel laser fournit en effet un train cohérent d'impulsions dont le spectre de Fourier est un peigne constitué par des modes distants les uns des autres de $\Omega=2 \pi / \tau$; où $\tau$ est le délai qui sépare deux impulsions successives. Lorsqu'on utilise ce laser pour effectuer une expérience de spectroscopie de saturation (Absorption Saturée [1] ou Spectroscopie de polarisation [2]), chaque résonance de saturation devient un peigne de résonances distantes de $\Omega / 2$.

Ce type d'expérience démontre qu'il est possible d'utiliser plusieurs classes de vitesses en spectroscopie de saturation à haute résolution avec un laser fonctionnant en impulsions. On retrouve en outre l'avantage d'une calibration précise de l'échelle des fréquences démontré antérieurement dans des expériences de spectroscopie à deux photons [3].

Cet article a trait à l'extension de cette méthode au cas d'une expérience d'absorption saturée avec un laser monomode continu. Une expérience de ce 
type a déjà été effectuée [4], elle mettait en œuvre un laser à $\mathrm{Ar}^{+}$monomode de largeur spectrale $2 \mathrm{MHz}$ et un montage d'absorption saturée classique. Nous proposons d'exploiter pleinement les avantages que peut apporter cette technique en l'associant à la détection hétérodyne par modulation de fréquence du faisceau saturant [9] et en utilisant un laser monomode de haute pureté spectrale [6].

Le principe consiste à réaliser un peigne de fréquences optiques centré autour de la fréquence $\omega_{\mathrm{L}}$ d'un faisceau laser monomode. Ce peigne dont les composantes adjacentes sont distantes de $\Omega$ peut être obtenu par modulation de phase, de fréquence ou d'amplitude. Il est ensuite utilisé dans une expérience de spectroscopie de saturation hétérodyne. Chaque résonance de saturation se décompose alors en un peigne de résonances dont les composantes adjacentes sont distantes de $\Omega / 2$. Pour résoudre ces résonances il est essentiel que l'écart de fréquence $\Omega$ entre les composantes adjacentes du peigne de fréquences optiques soit nettement supérieur à la largeur de raie.

Les expériences ont été effectuées sur les premières raies de la transition 43-0 $\mathrm{P}(13)$ de l'iode qui coïncident avec la longueur d'onde d'émission du laser à $\mathrm{Ar}^{+}$à $514,5 \mathrm{~nm}$. La largeur naturelle de ces raies est d'environ $70 \mathrm{kHz}$, mais dans les conditions de pression d'iode où nous avons opéré $(10 \mathrm{mT})$ l'élargissement lié aux collisions est de l'ordre de $600 \mathrm{kHz}$. Cela impose l'utilisation de fréquences de modulation élevées $(\Omega>1,2 \mathrm{MHz})$.

Le peigne de fréquences optiques a été réalisé par modulation de phase du faisceau de sortie du laser au moyen d'un cristal électro-optique, car cette technique est la mieux adaptée à ces gammes de fréquences. Son avantage majeur est qu'elle n'altère pas les caractéristiques géométriques du faisceau (fronts d'onde et direction de propagation), tout en permettant d'obtenir des indices de modulation suffisants pour la démonstration de notre méthode. Pour des fréquences de modulation plus basses, on pourrait utiliser des céramiques piézo-électriques ou des modulateurs acousto-optiques.

Le schéma du montage expérimental que nous avons mis en œuvre est donné en figure 1 . Nous avons utilisé

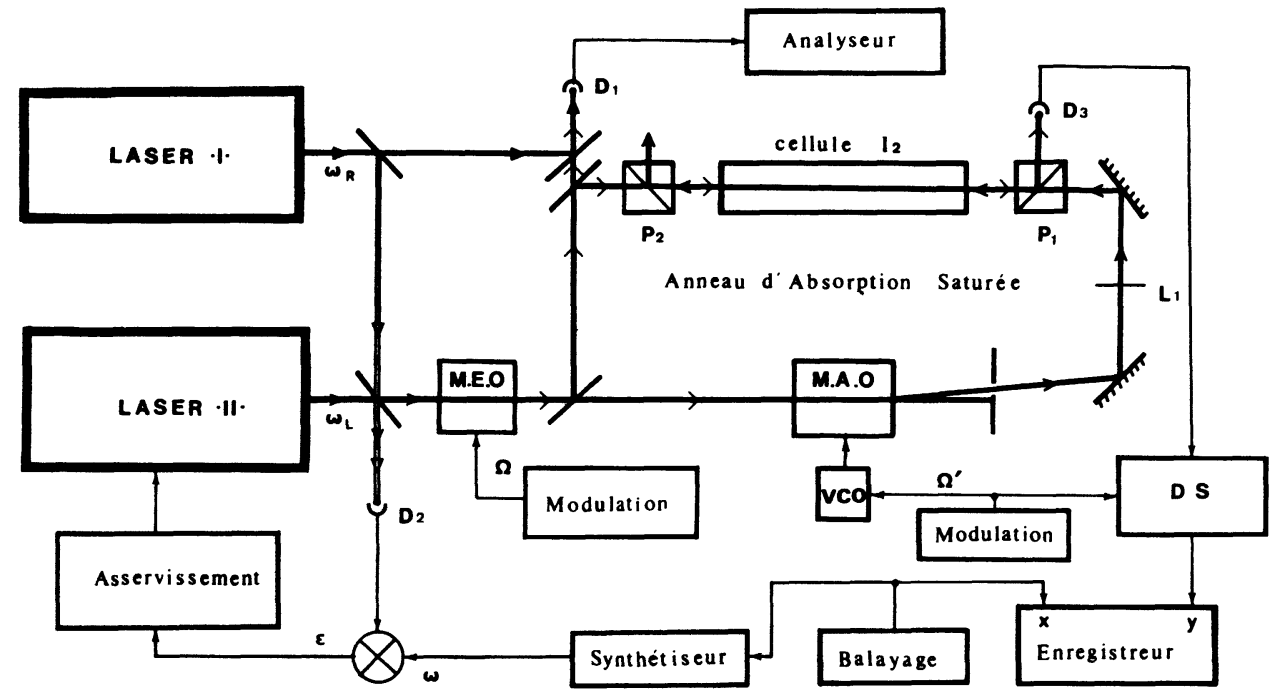

Fig. 1. - Schéma de principe du montage expérimental.

Le laser I stabilisé sur la raie $a_{3}$ de l'iode fournit une fréquence de référence $\omega_{R}$.

Le laser II préstabilisé sur Fabry-Perot, est asservi sur la référence $\omega_{\mathrm{R}}$ avec un décalage réglable $\omega$. Le signal d'erreur $\varepsilon$ utilisé dans cette boucle d'asservissement, résulte du mélange du signal de battement entre les deux lasers détecté par $D_{2}$ avec le signal de fréquence $\omega$ fourni par un synthétiseur balayable. Le laser II fournit ainsi un faisceau de fréquence $\omega_{\mathrm{L}}$ balayable et possédant les caractéristiques de stabilité de la référence $\omega_{R}$. Ce faisceau est ensuite modulé en phase à la fréquence $\Omega$ par le modulateur électro-optique (M.E.O.). L'analyse du signal de battement détecté par $\mathrm{D}_{1}$ permet de contrôler l'indice de modulation obtenu.

Le faisceau modulé pénètre ensuite dans l'anneau d'absorption saturée où le modulateur acousto-optique (M.A.O.) alimenté par un oscillateur contrôlé en tension (V.C.O.) a pour rôle de défléchir le faisceau saturant, de le décaler en fréquence de la quantité $\Delta$ et de le moduler en fréquence à la fréquence $\Omega^{\prime}$. La modulation à la fréquence $\Omega^{\prime}$ induite sur le faisceau sonde est détectée à l'aide d'une détection synchrone (D.S.).

[Experimental set-up.

The reference frequency $\omega_{R}$ is given by laser I stabilized on the iodine $a_{3}$ line.

Laser II is locked with a tunable frequency offset $\omega$ with respect to $\omega_{R}$. The frequency beat note of the lasers (given by $D_{2}$ ), after mixing with a signal at the tunable frequency $\omega$ delivered by a synthesizer gives the error signal $\varepsilon$. This method provides both stability and tunability to the $\omega_{\mathbf{L}}$ laser II beam. This beam is then phase-modulated at $\Omega$ by means of an electro-optic modulator (M.E.O.). The modulation index is monitored by a spectral analysis of the beat note detected by $D_{1}$.

The modulated beam enters a saturated absorption ring. The saturation beam is deflected, frequency shifted by $\Delta$ and frequency modulated at $\Omega^{\prime}$ by means of an acousto-optic modulator (M.A.O.) driven by a V.C.O. The induced $\Omega^{\prime}$ modulation is then detected by a lock-in (D.S.).] 
un laser à argon ionisé monomode, préstabilisé en fréquence sur le pic de transmission d'un résonateur de Fabry-Pérot extérieur très stable [6]. Le faisceau du laser préstabilisé traverse un cristal électro-optique dont l'axe extraordinaire est placé parallèlement au champ électrique. Lorsqu'on module à la fréquence $\Omega$ la différence de potentiel appliquée aux bornes du cristal, on module aussi le chemin optique parcouru par l'onde laser. A la sortie du cristal cette dernière est donc modulée en phase $[7,8]$ et son champ électrique s'écrit :

$$
E=\frac{1}{2} \varepsilon \exp \left\{i\left(\omega_{\mathrm{L}} t+\Phi_{0} \sin \Omega t+k z+\Phi\right)\right\}+\text { c.c. }
$$

Ce champ peut être décomposé en série de Fourier : $E=\frac{1}{2} \varepsilon \sum_{n=-\infty}^{+\infty} J_{n}\left(\Phi_{0}\right) \exp \left\{i\left[\left(\omega_{\mathrm{L}}+n \Omega\right) t+k z+\Phi\right]\right\}+$ c.c.

où $\Phi_{0}$ est l'indice de modulation et $J_{n}\left(\Phi_{0}\right)$ la fonction de Bessel de première espèce, d'ordre $n$ et d'argument $\Phi_{0}$.

En l'absence de modulation, la totalité de la puissance du laser est à la fréquence $\omega_{\mathbf{L}}$. Dès qu'on applique la modulation $\Omega$ aux bornes du cristal, cette puissance est répartie entre les différentes composantes de fréquences $\omega_{n}=\omega_{\mathrm{L}}+n \Omega$ qui constituent le spectre de la relation (2).

A la sortie du modulateur électro-optique, le faisceau laser modulé en phase est envoyé dans un anneau d'absorption saturée. On utilise la méthode de détection décrite dans les références $[6,9]$. La fréquence du faisceau saturant est décalée de la quantité $\Delta$ (ce qui permet d'obtenir une excellente isolation optique [10]) et modulée en fréquence à $\Omega^{\prime}$.

On détecte alors la composante de fréquence $\Omega^{\prime}$ de la modulation d'amplitude induite sur le faisceau sonde.

L'expression du champ électrique de l'onde sonde à l'entrée de la cellule est obtenue directement à partir de la relation (2).

Au contraire le champ saturant est soumis à une double modulation à $\Omega$ et à $\Omega^{\prime}$. Chaque composante du spectre donné par la relation (2) est décalée de $\Delta$ et modulée à la fréquence $\Omega^{\prime}$, d'où l'expression du champ saturant :

$$
\begin{aligned}
E_{\text {sat }}=\frac{1}{2} \varepsilon_{\text {sat }} & \sum_{n=-\infty}^{+\infty} J_{n}\left(\Phi_{0}\right) \exp \left\{i \left[\left(\omega_{\mathrm{L}}+n \Omega\right) t+\Delta t+\right.\right. \\
& \left.\left.+\frac{\delta^{\prime}}{\Omega^{\prime}} \sin \Omega^{\prime} t-k z+\Phi_{+}\right]\right\}+ \text {c.c. }
\end{aligned}
$$

Après développement en série de Fourier, on obtient :

$$
\begin{aligned}
E_{\text {sat }} & =\frac{1}{2} \varepsilon_{\text {sat }} \sum_{n^{\prime}=-\infty}^{+\infty} \sum_{n=-\infty}^{+\infty} J_{n}\left(\Phi_{0}\right) J_{n^{\prime}}\left(\frac{\delta^{\prime}}{\Omega^{\prime}}\right) \times \\
\times & \exp \left\{i\left[\left(\omega_{\mathbf{L}}+n \Omega+\Delta+n^{\prime} \Omega^{\prime}\right) t-k z+\Phi_{+}\right]\right\} \\
& + \text {c.c. }
\end{aligned}
$$

Dans ces expressions :

- $\frac{\delta^{\prime}}{\Omega^{\prime}}$ représente l'indice de modulation de fréquence à $\Omega^{\prime}$.

- On a choisi un champ saturant se propageant dans la direction des $z>0$.

La lame $\lambda / 2\left(\mathrm{~L}_{1}\right)$ associée au prisme de Glan $P_{1}$ permet de régler de façon continue l'intensité du faisceau saturant. La photodiode $\mathrm{D}_{1}$ détecte le battement entre le faisceau modulé en phase et le faisceau d'un laser de référence. L'analyse spectrale de ce battement permet de contrôler en permanence l'amplitude des diverses composantes $J_{n}\left(\Phi_{0}\right)$. Pour analyser commodément les formes de raies obtenues nous avons fait appel à la technique habituelle de décalage de fréquence [5]. Le laser I de référence étant asservi sur la raie $a_{3}$ de la transition 43-0 P (13), nous avons analysé la raie $\mathrm{a}_{4}$ avec le laser II balayable.

\section{Résultats expérimentaux.}

Nous avons utilisé le montage précédemment décrit pour étudier les formes de raies obtenues grâce à cette méthode. Nous avons enregistré l'harmonique 1 du signal d'absorption saturée détecté par la méthode de modulation de fréquence du faisceau saturant décrite dans les références $[6,9]$.

La figure 2 donne des exemples de formes de raies obtenues en présence et en absence de modulation de phase du faisceau laser, pour différentes puissances du faisceau saturant. Afin de pouvoir étudier commodément l'effet de la modulation de phase, nous avons effectué pour chacune des différentes valeurs $P_{1}, P_{2} \ldots P_{n}$ de la puissance du faisceau saturant, deux enregistrements successifs : un premier, en l'absence de modulation de phase pour une puissance $P_{i}$ du faisceau saturant; un deuxième, en présence de modulation de phase en réglant la puissance de la composante centrale du spectre du faisceau saturant à la même valeur $\boldsymbol{P}_{i}$. Les puissances des deux premières bandes latérales, respectivement proportionnelles à $J_{1}^{2}$ et $J_{-1}^{2}$ ont été réglées à $P_{i} / 2$, ce qui correspond à un indice de modulation $\Phi_{0} \simeq 1,15$.

Avec cet indice de modulation, la valeur des coefficients $J_{n}$ décroît rapidement avec $n$.

Ces enregistrements font apparaître que l'amplitude pic à pic de la raie centrale du peigne de résonances de saturation obtenue avec la modulation de phase est sensiblement plus grande que l'amplitude pic à pic de la raie obtenue sans modulation. Cette augmentation provient de la présence des bandes latérales dans le spectre du faisceau laser à l'entrée de 


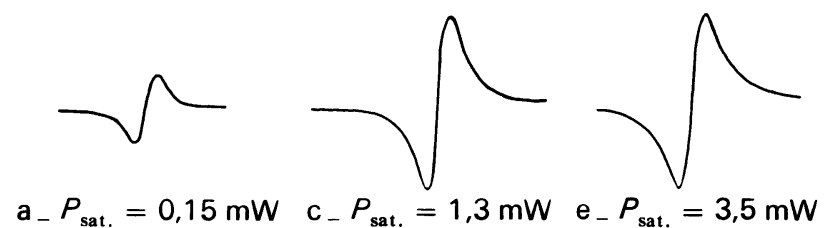

$$
a_{-} P_{\text {sat. }}=0,15 \mathrm{~mW} c_{-} P_{\text {sat. }}=1,3 \mathrm{~mW} e_{-} P_{\text {sat. }}=3,5 \mathrm{~mW}
$$

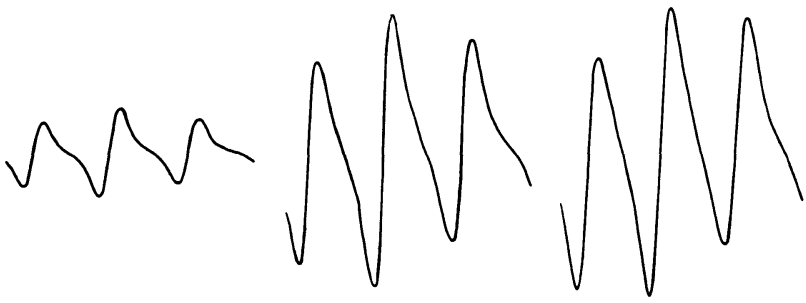

b_ $P_{\text {sat. }}=0,15 \mathrm{~mW} \mathrm{~d}_{-} P_{\text {sat. }}=1,3 \mathrm{~mW} \quad \mathrm{f}_{-} P_{\text {sat. }}=3,5 \mathrm{~mW}$

Fig. 2. - Exemples de formes de raies obtenues pour différentes puissances du faisceau saturant.

On a opéré à puissance constante du faisceau sonde $\left(P_{\text {sond. }}=0,25 \mathrm{~mW}\right)$ et avec des diamètres sensiblement identiques pour les deux faisceaux $\left(w_{0}=0,86 \mathrm{~mm}\right)$. Les fréquences de modulation étaient : $\Omega \simeq 4,4 \mathrm{MHz}$ et $\Omega^{\prime} \simeq 60 \mathrm{kHz}$.

Les courbes a, c, e ont été obtenues sans modulation de phase; les courbes b, d, f ont été obtenues avec modulation de phase. Pour les courbes b, d, f; $P_{\text {sat. }}$ désigne la puissance de la composante centrale du spectre du faisceau saturant.

[Line shapes observed for various saturating beam powers : The probe beam power ..... was $P_{\text {sond. }} \simeq 0.25 \mathrm{~mW}$, the waist of each beam ..... was ... $w_{0} \simeq 0.86 \mathrm{~mm}$, the modulation frequencies... were $\ldots \Omega \simeq 4.4 \mathrm{MHz}$, and $\Omega^{\prime} \simeq 60 \mathrm{kHz}$.

a, c, e curves were obtained without phase-modulation and $\mathrm{b}, \mathrm{d}, \mathrm{f}$ ones with phase modulation. In the last case, $P_{\text {sat. }}$ is the power of the central component of the saturating beam spectrum.]

l'anneau de saturation. Elle s'explique par l'interaction simultanée des composantes de l'onde sonde de fréquences $\omega_{\mathrm{L}} \pm n \Omega$, et des composantes de l'onde saturante de fréquences $\omega_{\mathrm{L}} \mp n \Omega$, avec les molécules. Cette interaction est résonnante pour la fréquence $\omega_{0}$ de la transition et elle fait intervenir des molécules appartenant à des classes de vitesses différentes de zéro : $v_{z}= \pm n \Omega / k$.

Les courbes des figures 3 et 4 ont été obtenues à partir du dépouillement d'une série d'enregistrements du type précédent.

La figure 3 montre comment évolue l'amplitude pic à pic de l'harmonique 1 de la raie $\mathrm{a}_{4}$ lorsqu'on change la puissance du faisceau saturant, en présence de modulation de phase (Fig. 3b) et sans modulation de phase (Fig. 3a). Ces deux courbes confirment ce qui précède; on observe une augmentation de l'amplitude d'un facteur 1,6 pour des puissances du faisceau saturant d'environ $2,5 \mathrm{~mW}$. On constate en outre que ces courbes présentent un maximum, ceci peut s'interpréter par l'influence de l'élargissement par saturation aux fortes puissances, car l'amplitude de l'harmonique 1 dépend de la largeur de raie.

Les courbes de la figure 4 montrent l'évolution de

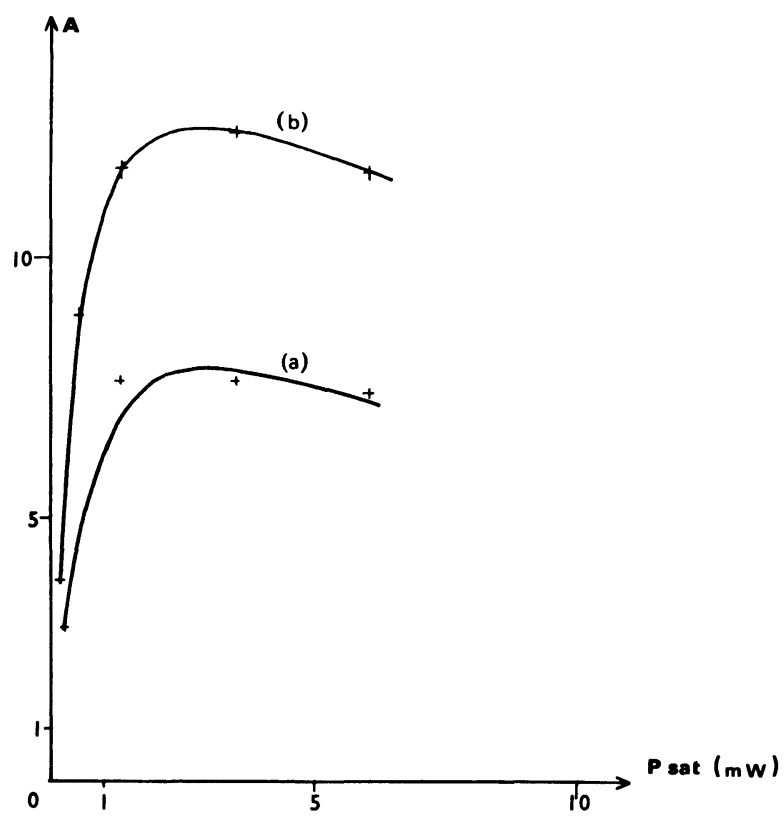

Fig. 3. - Amplitude pic à pic (A) en unité arbitraire de l'harmonique 1 de la raie $a_{4}$ de l'iode en fonction de la puissance du faisceau saturant. a) Sans modulation de phase. b) Avec modulation de phase.

[Peak to peak amplitude (A) in arbitrary units of iodine $a_{4}$ line first harmonic versus saturating beam power : a) without phase-modulation, b) with phase-modulation.]

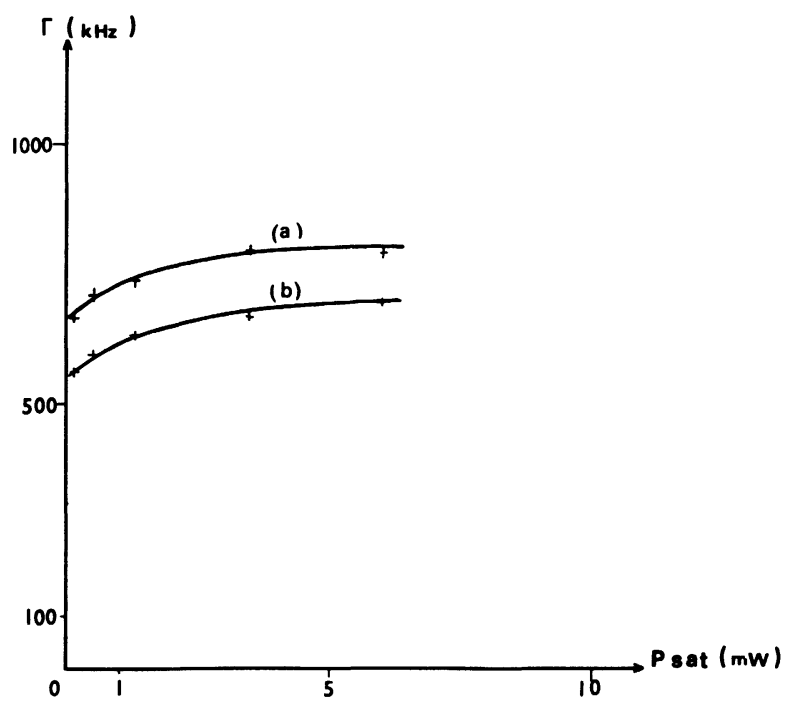

Fig. 4. - Largeur pic à pic $(\Gamma)$ de l'harmonique 1 de la raie $a_{4}$ de l'iode en fonction de la puissance du faisceau saturant : a) Sans modulation de phase, b) Avec modulation de phase.

[Peak to peak width $(\Gamma)$ of iodine $a_{4}$ line first harmonic versus saturating beam power : a) without phase-modulation, b) with phase-modulation.]

la largeur pic à pic de l'harmonique 1 de la raie $\mathrm{a}_{4}$ en fonction de la puissance du faisceau saturant. On remarque que la largeur de la raie centrale obtenue 
lorsque le faisceau laser est modulé en phase est plus faible que celle de la raie détectée en absence de modulation. Ceci peut s'interpréter qualitativement de la manière suivante : les puissances des bandes latérales du faisceau laser modulé en phase ayant été réglées dans tous les cas à des valeurs plus faibles que celle de la composante centrale $\boldsymbol{P}_{i}$, les classes de molécules de vitesses $v_{z} \neq 0$ qui viennent contribuer au signal voient un champ saturant plus faible que celui vu par la classe de molécules de vitesse $v_{z}=0$. Il en résulte que la contribution au signal provenant des molécules de vitesses $v_{z} \neq 0$ est moins élargie par saturation que celle qui provient des molécules de vitesse $v_{z}=0$.

Les résultats qui précèdent montrent que la sensibilité des méthodes habituellement utilisées en spectroscopie de saturation (absorption saturée, dispersion saturée, procédés de détection H.F. et hétérodyne divers, spectroscopie de polarisation...) peut être améliorée grâce à la contribution de plusieurs classes de vitesses au signal de saturation. En contrepartie, c'est grâce à la mise en œuvre des méthodes de détection hétérodyne de haute sensibilité et à l'utilisation d'un laser de haute pureté spectrale qu'il a été possible de mettre clairement en évidence les avantages de cette technique. Comme le procédé n'introduit pas de bruit propre, l'augmentation de l'amplitude du signal associée à une réduction de l'élargissement par saturation se traduit bien par une augmentation notable du rapport signal/bruit.

On pourrait obtenir une augmentation beaucoup plus spectaculaire de la sensibilité en augmentant l'indice de modulation. La limite à ne pas dépasser est celle où le peigne de fréquences optiques s'étend sur toute la largeur Doppler. Dans le cas de la transition de l'iode sur laquelle ont porté nos expériences le gain pourrait être très important puisque le rapport entre la largeur Doppler et la largeur de raie expérimentale est de l'ordre de $10^{3}$, ce qui autorise l'utilisation d'un peigne comprenant un très grand nombre de composantes. Cependant, en pratique, l'existence de raies voisines impose une limitation plus étroite que celle de la largeur Doppler. A cet égard, les premières raies de la transition 43-0 P (13) sont les plus favorables car elles sont les mieux isolées du spectre.

Cette méthode peut donc trouver un large champ d'applications pour les étalons de fréquence et la spectroscopie sub-Doppler à très haute résolution. En particulier, elle vient d'être appliquée avec succès dans notre laboratoire dans des expériences d'absorption saturée à très haute résolution à l'aide de lasers à $\mathrm{CO}_{2}$ dans la région spectrale de $10 \mu \mathrm{m}$ [11]. Les très faibles largeurs de raies ( 1 à $2 \mathrm{kHz}$ ) obtenues avec des molécules lourdes comme $\mathrm{SF}_{6}$ et $\mathrm{OsO}_{4}$ permettent d'utiliser des fréquences de modulation beaucoup plus basses et des indices de modulation plus élevés que dans le visible, ce qui permet d'exploiter pleinement les possibilités offertes par cette méthode.

\section{Remerciementș.}

Les auteurs expriment leur reconnaissance à MM. Ch. J. Bordé, J. P. Descoubes et M. Ducloy pour l'intérêt qu'ils ont porté à ce travail.

\section{Bibliographie}

[1] Couillaud, B., Ducasse, A., SARger, L. and Boscher, D., Appl. Phys. Lett. 36 № 6 (1980) 407-409.

[2] Ferguson, A. I., Eckstein, J. N. and HänSCH, T. W., Appl. Phys. 18 (1979) 257-260.

[3] Eckstein, J. N., Ferguson, A. I., HäNSCh, T. W., Phys. Rev. Lett. 40 (1978) 847-850.

Salour, M. M. and Cohen-Tannoudj, C., Phys. Rev. Lett. 38 (1977) 757.

[4] Kramer, G., Ghosh Roy, D. N., Helmcke, J. and SPIEWECK, F., Appl. Phys. Lett. 37 (1980) 354-356.

[5] Bordé, Ch. J., Camy, G., Decomps, B. et Descoubes, J. P., J. Physique 42 (1981) 1393-1411.
[6] Camy, G., Pinaud, D., Courtier, N. et Hu Quiquan, Revue Phys. Appl. 17 (1982) 357-363.

[7] YARIV, A., Introduction to optical Electronics, Copyright by Holt Rinehart and Winston (1971) p. 236.

[8] Hall, J. L., Hollberg, L., Baer, T. and Robinson, H. G., Appl. Phys. Lett. 39 (1981) 680-682.

[9] Camy, G., Bordé, Ch. J. and DuCloy, M., Optics Commun. 41 (1982) 325-330.

[10] SNyder, J. J., RAJ, R. K., Bloch, D. and Ducloy, M., Opt. Lett. 5 (1980) 1963.

[11] Salomon, Ch., Camy, G., Vanlerbergue, A. and BoRDÉ, Ch. J., à paraître. 